\title{
Las habilidades sociales y el uso de redes sociales virtuales en estudiantes universitarios de Lima Metropolitana ${ }^{1}$
}

Ana Esther Delgado

Luis Escurra

María Clotilde Atalaya Juan Pequeña Constantino Abel Cuzcano

Rosa Emilia Rodríguez David Álvarez

Universidad Nacional Mayor de San Marcos

Recibido: 19 de abril del 2016 / Aprobado: 16 de junio del 2016

Se estudió la relación entre las habilidades sociales y el uso de redes sociales virtuales en estudiantes de una universidad pública y una particular de Lima Metropolitana. Se trabajó con una muestra de 1405 estudiantes universitarios, obtenida a través de un muestreo no probabilístico de tipo intencionado. Los resultados observados indican que existen correlaciones negativas y estadísticamente significativas entre los puntajes totales de la Escala de Habilidades Sociales y los del Cuestionario de Adicción a las Redes Sociales, así como en la mayoría de los factores de ambos instrumentos.

habilidades sociales / uso de redes sociales virtuales / estudiantes universitarios

\section{Social Skills and Use of Virtual Social Networks in University Students in Metropolitan Lima}

We studied the relationship between social skills and use of virtual social networks in students of a public university and a private one in the city of Lima. The sample were 1405 university students and it was an intentional non-probability type. The results indicate that there are negative and statistically significant correlations between total scale scores on social skills and addiction questionnaire to social networks, and in most of the factors of both instruments.

social skills / virtual social networks / university students

Correo electrónico: aedvdt2@yahoo.com

1 El estudio fue patrocinado por el Vicerrectorado de Investigación y el Consejo Superior de Investigaciones (CSI) de la UNMSM, código n. ${ }^{\circ}$ 151801031, el año 2015. Colaboraron en el estudio los estudiantes de pregrado Carla Lidia Espejo López, Bill Kevin Egúsquiza Vásquez, Fiorella Litzi Verde Guere y María del Carmen Ayala Bellido. 


\section{INTRODUCCIÓN}

Gismero (2000) considera que la conducta socialmente habilidosa es aquella que incluye un conjunto de respuestas verbales y no verbales específicas para el escenario social en el que se vive, las cuales están relacionadas con las necesidades, los sentimientos, las opiniones y preferencias expresadas sin muestras de ansiedad y de manera no aversiva, teniendo respeto mutuo.

Lucena, Fernández y Francisco (2002) señalan que existen diversas definiciones de las habilidades sociales, pero que se puede observar un común denominador, debido a que se las considera como un conjunto de comportamientos en las relaciones interpersonales que resultan eficaces, y tienen dos objetivos principales, los cuales son afectivos (tener relaciones satisfactorias con parientes, amigos y con la pareja) e instrumentales (realizar con éxito actividades en la comunidad, como uso de servicios, entrevistas satisfactorias de trabajo, realizar adecuadamente transacciones de compra y venta, etc.).

Las habilidades sociales son parte importante de la construcción de la identidad de las personas a través del compartir con pares, y reflejan distintas formas de comunicarse mediante gestos, tono de voz, atuendos, postura, etc. El aprendizaje adquirido en la familia se complementa posteriormente con las influencias que se reciben en el grupo social de la escuela, del vecindario, etc. (Shaffer y Kipp, 2007).
Las habilidades sociales son aprendidas y, según Ballesteros y Gil (2002), dependen de los siguientes factores: a) un modelo adecuado que permite aprender cómo resolver conflictos mediante el diálogo, el desarrollo de conductas asertivas, etc.; b) una valoración permanente de los aspectos positivos de la conducta humana; c) facilitar el desarrollo del pensamiento que promueva la búsqueda múltiple de alternativas a la solución de un determinado problema; d) facilitar momentos u ocasiones para el desarrollo de las habilidades sociales en contextos de actuación (ficticios) fáciles de relacionar a la hora de llevarlos a cabo en una situación real. Una persona con un buen desarrollo de su habilidad social, generalmente, ha tenido como referente un buen modelo a seguir, el cual la ayudará a potenciar su desarrollo y bienestar social.

\section{Redes sociales}

La estructura de los roles sociales, dentro de un contexto más amplio al que representó la familia en un primer momento, en los escolares, y posteriormente en los universitarios, demanda mayor participación e involucramiento del individuo, que se enfrenta a nuevos escenarios sociales de interacción (Galarcio, 2006).

La teoría de las redes ha sido desarrollada desde diferentes enfoques, pensamientos y teorías, como las matemáticas, la psicología y sociología, entre otras. Lozarez (1996) define las redes sociales como un conjunto bien delimitado de individuos, grupos, organizaciones, comunidades, sociedades globales, entre 
otros, vinculados unos a otros a través de una relación o conjunto de relaciones. En la actualidad, dicho concepto ha sido modificado debido a la presencia de las nuevas tecnologías de la información, el Facebook, Twitter, WhatsApp, Tuenti, Google, entre otros sistemas de interacción social, de tal manera que el concepto ha derivado hacia ellos debido a los motivos por los que fueron desarrollados.

Con el desarrollo de internet y la posibilidad de mantener relaciones sociales a distancia, han logrado que el concepto de comunidad desaparezca (Fuente et al., 2010). Por su parte, Smith (2003, citado en Fuente et al., 2010) expone que las comunidades online se diferencian de otras en la medida que establecen límites cerrados, de manera que los usuarios pueden entrar y salir con un costo mínimo al que se produciría si se realizara el cambiar de una comunidad física a otra nueva. Una característica adicional de las redes online es el hecho de que la interacción es anónima, pues se realiza a través de una computadora, además de que las relaciones en las redes sociales son más débiles y susceptibles de disolverse, pues la idea de pertenecer a una comunidad online es fugaz si se la compara con las comunidades reales, con las redes cara a cara, que suelen ser densas.

Las redes sociales, dentro de los espacios de interacción social, constituyen una opción virtual, cuya proliferación en uso se ha incrementado en los últimos años (Meattle, 2007). Orihuela (2008) señala que estas permiten a sus usuarios establecer contacto mutuamente, compartir información, coordinar acciones, etc. La creciente proliferación de estos recursos ha generado un desplazamiento notorio y progresivo del contacto personal, lo cual puede afectar las relaciones interpersonales y generar que se vaya perdiendo la capacidad para interpretar el estado de ánimo de la otra persona, comprender su lenguaje corporal, y hasta ser asertivos en cuanto a dar opiniones a los demás; incluso se podría, en un futuro, optar por evitar el contacto personal (Carballo, Pérez-Jover, Espada, Orgilés, y Piqueras, 2012).

Según Carballo et al. (2012), autores como Caplan, Engelberb, Sjöberg, Echerburtía, Labrador y Becoña señalan que, con el creciente uso de las redes sociales virtuales, se ha producido un cambio vertiginoso en el sistema de relaciones sociales que no ha sido acompañado con investigaciones científicas que permitan su comprensión y, por tanto, un mejor manejo de estos avances en las comunicaciones interpersonales, lo cual ha limitado el desarrollo de estrategias educativas y preventivas en el manejo de este tipo de herramientas virtuales, y más aún en considerar las consecuencias de su uso inadecuado. Algunas investigaciones ofrecen evidencias de repercusiones negativas que se han venido presentando, como el incremento del aislamiento social, la depresión, el inadecuado ajuste social, la adicción a las redes sociales, el abuso de internet, etc.

Entre las investigaciones internacionales desarrolladas, se reportan las siguientes. 
Zywica y Danowski (2008) llevaron a cabo un estudio sobre la apariencia social de los usuarios de una red social muy conocida, basándose en la popularidad fuera de líneas relacionada con la sociabilidad y la estima personal. Los resultados mostraron que el subconjunto de usuarios que se acercaban a perfiles extrovertidos y con mayor estima personal apoyaron la hipótesis de mejoramiento social, y eran más populares en la red social y fuera de ella. En cambio, el grupo de usuarios con perfiles introvertidos y con menos estima personal mostraban mayor esfuerzo para verse más populares en esta red social.

Por otro lado, Subrahmanyan y Greenfield (2008) revelaron en los resultados de su estudio que los adolescentes que usaban herramientas de comunicación online paralelamente a su entorno social físico reforzaban las relaciones con amigos; además, disminuía la ansiedad social frente a personas de sexo diferente. Las consecuencias de este tipo de interacción se manifestaban de manera positiva y negativa en la vida de los adolescentes; eran positivas por los beneficios de la interacción libre y dinámica entre los usuarios, pero podían ser negativas porque los adolescentes se encontraban expuestos a mensajes con diversas connotaciones negativas; por ejemplo, racismo, violencia, entre otras.

Valkenburg y Peter (2008) estudiaron la relación entre el uso frecuente de internet con las competencias sociales que establecían los adolescentes fuera del área virtual y dentro de ella, además de ver qué relación guardaban con la experiencia de soledad y el autoconcepto. Los resultados mostraron que la comunicación a través de internet contribuyó de manera positiva a las competencias sociales de los usuarios y no afectó la unidad del autoconcepto.

García del Castillo, Terol, Nieto, Lledo, Sánchez, Aragón y Sitges (2008) llevaron cabo una investigación acerca del uso y abuso de internet en 391 estudiantes de la Universidad Miguel Hernández en Alicante, España. Se encontró que la mayoría de los participantes había utilizado alguna vez internet, y fueron los estudiantes de Psicología quienes presentaron un mayor porcentaje de uso varias veces al día. Asimismo, gran parte de los participantes señaló que pasaba más de dos horas conectado a internet (utilizando el correo electrónico, el chat o messenger). El grupo de estudiantes con menor frecuencia de uso de internet presentó un déficit menor en el componente de las habilidades sociales frente al grupo de mayor frecuencia de uso (varias veces al día, entre 5 a 7 veces por semana).

Herrera, Pacheco, Palomar y Zavala (2010) investigaron la relación entre la adicción a Facebook, la baja autoestima, la depresión y la falta de habilidades sociales en 60 estudiantes universitarios de la Universidad Iberoamericana de México. Los resultados mostraron que los participantes adictos a Facebook eran más propensos a la depresión, eran menos asertivos, tenían menor calidad en sus relaciones familiares y amicales, tenían más dificultades para hablar en público que aquellos participantes que no eran 
adictos. Asimismo, mostraron una mayor tendencia a tener baja autoestima y presentaban una percepción más devaluada de su físico, su autoconcepto e intelecto.

En el Perú, Mejía, Paz y Quinteros (2014) estudiaron la relación entre la dependencia a Facebook y las habilidades sociales en estudiantes de una universidad privada de Lima. Participaron 300 estudiantes; utilizaron el Test de Adicción a Internet de Young, adaptado y validado por Mariani en 2005, y la Escala de Habilidades Sociales de Gismero. Se encontró una correlación inversa y significativa entre la dependencia a Facebook y las habilidades sociales de los estudiantes.

Escurra y Salas (2014) diseñaron, construyeron y validaron el Cuestionario de Adicción a Redes Sociales (ARS) en 380 estudiantes de diferentes universidades de la ciudad de Lima. Los resultados indicaron que existían tres dimensiones que se analizaron de forma independiente. Las funciones de información de los ítems evidenciaron que las dimensiones eran más precisas para discriminar a los individuos con niveles medios y altos del rasgo evaluado. Los resultados revelaron que la escala y sus componentes presentaron adecuadas propiedades psicométricas de validez y confiabilidad.

\section{OBjetIVOS}

\section{Objetivo general}

Analizar la relación entre las habilidades sociales y el uso de redes sociales virtuales en estudiantes universitarios de una universidad pública y una particular de Lima Metropolitana

\section{Objetivos específicos}

1. Identificar las habilidades sociales de los estudiantes universitarios de una universidad pública y una particular de Lima Metropolitana

2. Identificar el uso de las redes sociales virtuales en estudiantes universitarios de una universidad pública y una particular de Lima Metropolitana

3. Conocer la relación entre el factor autoexpresión en situaciones sociales y el factor obsesión por las redes sociales en los estudiantes universitarios de una universidad pública y una particular de Lima Metropolitana

4. Conocer la relación entre el factor autoexpresión en situaciones sociales y el factor falta de control personal en el uso de las redes sociales en los estudiantes universitarios de una universidad pública y una particular de Lima Metropolitana

5. Conocer la relación entre el factor autoexpresión en situaciones sociales y el factor uso excesivo de las redes sociales en los participantes

6. Conocer la relación entre el factor defensa de los propios derechos como consumidor y el factor obsesión por las redes sociales en los participantes

7. Conocer la relación entre el factor defensa de los propios derechos como consumidor y el factor falta de control personal en el uso de las redes sociales 
8. Conocer la relación entre el factor defensa de los propios derechos como consumidor y el factor uso excesivo de las redes sociales

9. Conocer la relación entre el factor expresión de enfado o disconformidad y el factor obsesión por las redes sociales en los participantes

10. Conocer la relación entre el factor expresión de enfado o disconformidad y el factor falta de control personal en el uso de las redes sociales

11. Conocer la relación entre el factor expresión de enfado o disconformidad y el factor uso excesivo de las redes sociales

12. Conocer la relación entre el factor $d e-$ cir "no" y cortar interacciones y el factor obsesión por las redes sociales

13. Conocer la relación entre el factor decir "no" y cortar interacciones y el factor falta de control personal en el uso de las redes sociales

14. Conocer la relación entre el factor decir "no" y cortar interacciones y el factor uso excesivo de las redes sociales

15. Conocer la relación entre el factor $h a-$ cer peticiones e iniciar interacciones positivas con el sexo opuesto y el factor obsesión por las redes sociales

16. Conocer la relación entre el factor $h a-$ cer peticiones e iniciar interacciones positivas con el sexo opuesto y el factor falta de control personal en el uso de las redes sociales

17. Conocer la relación entre el factor $h a-$ cer peticiones e iniciar interacciones positivas con el sexo opuesto y el factor uso excesivo de las redes sociales en los estudiantes universitarios de una universidad pública y una particular de Lima Metropolitana

\section{Método}

Se trata de un estudio de tipo descriptivo, en el que se utilizó un diseño de carácter correlacional, pues se trata de determinar el grado de relación existente entre dos variables, en este caso, la relación entre las habilidades sociales y el uso de redes sociales en estudiantes procedentes de una universidad pública y una particular de Lima Metropolitana (Alarcón, 2008; Sánchez y Reyes, 2015).

\section{Participantes}

El universo de investigación estuvo conformado por los universitarios matriculados en diversas especialidades de una universidad pública y una particular de Lima Metropolitana. El tamaño de la muestra se determinó tomando en cuenta las características de la población. Para la obtención de la muestra, se aplicó un procedimiento de muestreo no probabilístico de tipo intencionado, se seleccionaron las instituciones a ser incluidas en el estudio, y posteriormente en cada universidad se determinó el número de la muestra manteniendo la proporción de la población. Los participantes se seleccionaron de manera aleatoria. Participaron 1405 estudiantes universitarios.

Los instrumentos utilizados fueron los siguientes: 
1. Escala de Habilidades Sociales (EHS): constituye la versión mexicana de la escala elaborada por Ramírez (Godoy, 2010) a partir de la Escala de Habilidades Sociales de Gismero (2000), con 33 ítems.

La escala presenta los siguientes seis factores o dimensiones, obtenidos a través del análisis factorial:

a) Autoexpresión en situaciones sociales: se refiere a la capacidad de expresar opiniones, sentimientos, intereses, preguntas de manera espontánea y sin sentir ansiedad en diversas situaciones sociales (tiendas, grupos, reuniones sociales, entrevistas de trabajo, oficinas públicas o privadas, etc.).

b) Defensa de los propios derechos como consumidor: hace referencia a la conducta asertiva ante desconocidos con el objetivo de defender los derechos propios en situaciones de consumo, como no permitir que se adelanten en una fila, solicitar a alguien que guarde silencio, devolver objetos que no funcionan bien, etc.

c) Expresión de enfado o disconformidad: tiene que ver con la capacidad para enfrentar conflictos o confrontaciones con otras personas, como expresar enfado o sentimientos negativos justificados y desacuerdos con otras personas.

d) Decir "no"ycortarinteracciones:se refiere a la habilidad para finalizar interacciones que no se desean mantener, así como negarse a prestar algo o hacer algo cuando no se desea (favor o solicitud).

e) Hacer peticiones: corresponde a la capacidad para solicitar o pedir algo a otras personas sin excesiva dificultad, sea un amigo, un desconocido, o también en situaciones de consumo.

f) Iniciar interacciones positivas con el sexo opuesto: tiene que ver con la habilidad para iniciar conversaciones o pedir una cita; el poder ofrecer espontáneamente, y sin ansiedad, un halago, un cumplido; o el poder hablar con alguien que resulte atractivo.

La escala presenta evidencias de validez relacionadas con la estructura interna, así como una buena estimación de la confiabilidad por consistencia interna.

2. Cuestionario de Adicción a Redes Sociales (ARS): desarrollado por Escurra y Salas (2014) en el contexto limeño utilizando los criterios desarrollados por el DSM IV para el caso de las adiciones psicológicas. Está conformado por tres factores:

a) Obsesión por las redes sociales: se refiere al compromiso mental con las redes sociales, al estar pensando constantemente y fantaseando con ellas, a la ansiedad y la preocupación que causa el no poder acceder a las redes.

b) Falta de control personal en el uso de las redes sociales: tiene 
que ver con la preocupación por la falta de control o el no poder interrumpir el uso de las redes sociales, lo cual genera descuido de las tareas y los estudios.

c) Uso excesivo de las redes sociales: corresponde a las dificultades para poder controlar el uso de las redes sociales e indica un tiempo de uso excesivo; se refiere a la falta de capacidad para controlarse cuando se utilizan las redes sociales y no se puede disminuir la cantidad de tiempo de uso.

El instrumento fue desarrollado a partir de la teoría de la respuesta al ítem (TRI), sobre la base del modelo de Samejima (Escurra y Salas, 2014). Los autores reportaron que el instrumento presenta evidencias de validez relacionadas con el contenido y la estructura interna, así como una adecuada estimación de la confiabilidad por consistencia interna.

\section{Resultados}

Para determinar el estadístico a utilizar en el contraste de hipótesis, se aplicó la Prueba de Bondad de Ajuste a la curva normal de Shapiro Wilk, pues el estudio desarrollado por Razali y Wah (2011) ha demostrado que tiene mayor potencia estadística. Este estadístico fue aplicado a los puntajes de los factores y al total de la Escala de Habilidades Sociales (EHS), versión mexicana de Ramírez del 2008
(Godoy, 2010), y del Cuestionario de Adicción a las Redes Sociales (ARS), de Escurra y Salas (2014) (Cohen, 1988; Delgado, Escurra, y Torres, 2006; Siegel y Castellan, 2012). Los resultados de la Tabla 1 muestran valores de Shapiro-Wilk estadísticamente significativos tanto en los factores como en el puntaje total de la Escala de Habilidades Sociales (EHS) (autoexpresión en situaciones sociales: $\mathrm{W}=.062, \mathrm{p}<.001 ;$ defensa de los derechos: $\mathrm{W}=.121, \mathrm{p}<.001 ;$ expresión de enfado o disconformidad: $\mathrm{W}=.082, \mathrm{p}<.001$; decir "no" $y$ cortar interacciones: $\mathrm{W}=.087$, $\mathrm{p}<.001$; hacer peticiones: $\mathrm{W}=.107$, $\mathrm{p}<.001$; iniciar interacciones sociales positivas: $=.099, \mathrm{p}<.001, \mathrm{y}$ total de habilidades sociales: $\mathrm{W}=.048, \mathrm{p}<.001$ ), lo que indica que los puntajes no se distribuyen de acuerdo a la curva normal.

Asimismo, en los factores y el puntaje total del Cuestionario de Adicción a las Redes Sociales (ARS), se observan valores de Shapiro-Wilk que también son estadísticamente significativos (obsesión por las redes sociales: $\mathrm{W}=.109, \mathrm{p}<.001$; falta de control personal en el uso de las redes sociales: $\mathrm{W}=.081, \mathrm{p}<.001 ; \mathrm{y}$ uso excesivo de las redes sociales: $\mathrm{W}=.069$, $\mathrm{p}<.001$ ), lo cual significa que los puntajes no provienen de poblaciones que tengan una distribución normal.

Los resultados obtenidos en ambos instrumentos señalan que es necesario utilizar un estadístico no paramétrico para el contraste de hipótesis; en este caso, es la correlación Rho de Spearman. 
Tabla 1

Prueba de Bondad de Ajuste de Shapiro-Wilk de las pruebas evaluadas

\begin{tabular}{ll}
\hline Factores & W \\
\hline Autoexpresión en situaciones sociales & $.062^{* * *}$ \\
Defensa de los derechos & $.121^{* * *}$ \\
Expresión de enfado o disconformidad & $.082^{* * *}$ \\
Decir "no" y cortar interacciones & $.087^{* * *}$ \\
Hacer peticiones & $.107^{* * *}$ \\
Iniciar interacciones sociales positivas & $.099^{* * *}$ \\
\hline Total Escala de Habilidades Sociales & $.048^{* * *}$ \\
\hline Obsesión por las redes sociales & $.109^{* * *}$ \\
Falta de control personal en el uso de las redes sociales & $.081^{* * *}$ \\
Uso excesivo de las redes sociales & $.069^{* * *}$ \\
\hline Total Cuestionario de Adicción a las Redes Sociales & $.083^{* * *}$ \\
\hline
\end{tabular}

Nota: ${ }^{* *} \mathrm{p}<.001$

Se calculó el coeficiente de correlación Rho de Spearman entre el puntaje total de la Escala de Habilidades Sociales (EHS) y el puntaje total del Cuestionario de Adicción a las Redes Sociales (ARS) (Tabla 2), y se obtuvo un coeficiente Rho de Spearman de -.26, $\mathrm{p}<.001$, la cual es una correlación negativa y estadísticamente significativa. Este resultado valida la hipótesis general que plantea una relación negativa y estadísticamente significativa entre las habilidades sociales y el uso de redes sociales virtuales en estudiantes de una universidad pública y una privada de Lima Metropolitana.

\section{Tabla 2}

Coeficiente Rho de Spearman de los puntajes totales de la EHS y el ARS de los estudiantes de una universidad pública y una particular de Lima Metropolitana

\begin{tabular}{lc}
\hline \multicolumn{1}{c}{ Variable } & $\begin{array}{c}\text { Puntaje total del Cuestionario } \\
\text { de Adicción a las Redes } \\
\text { Sociales }\end{array}$ \\
\hline Puntaje total de la Escala de Habilidades Sociales & $-.26^{* * *}$ \\
\hline $\begin{array}{l}\text { Nota: } \mathrm{N}=1405 \\
* * * \mathrm{p}<.001\end{array}$ & \\
\hline
\end{tabular}


En la Tabla 3, se presentan los resultados del contraste de todas las hipótesis específicas. En los de la hipótesis específica $\mathrm{H}_{1.1}$, que plantea la existencia de una relación negativa y estadísticamente significativa entre el factor autoexpresión en situaciones sociales y el factor obsesión por las redes sociales, se puede observar que se obtiene una correlación negativa estadísticamente significativa ( $\mathrm{rho}=-.25$ $\mathrm{p}<.001$ ), que corresponde a un tamaño del efecto pequeño y a una potencia estadística $(1-\beta=1.00)$, lo cual permite concluir que se valida la hipótesis específica $\mathrm{H}_{1.1}$.

En cuanto a la hipótesis específica $\mathrm{H}_{1.2}$, que plantea la existencia de una relación negativa y estadísticamente significativa entre el factor autoexpresión en situaciones sociales y el factor falta de control personal en el uso de las redes sociales, en la Tabla 3, se observa un coeficiente de correlación estadísticamente significativo y negativo ( $r h o=-.25 \mathrm{p}<.001)$ con un tamaño del efecto pequeño. Este hallazgo permite señalar que se valida la hipótesis específica $\mathrm{H}_{1.2}$.

También se puede observar que, entre el factor autoexpresión en situaciones sociales y el factor uso excesivo de las redes sociales en los participantes, se obtiene un coeficiente estadísticamente significativo y negativo (rho=-.20 ( $\mathrm{p}<.001)$, que presenta un tamaño del efecto pequeño. Por tanto, se valida la hipótesis específica $\mathrm{H}_{1.3}$.

El resultado de la correlación Rho de Spearman entre los puntajes del factor defensa de los propios derechos como consumidor y el factor obsesión por las redes sociales en los estudiantes de una universidad pública y una particular de Lima Metropolitana permite apreciar un coeficiente de -.12 ( $\mathrm{p}<.001)$, lo que indica la existencia de una correlación negativa estadísticamente significativa, que presenta un tamaño del efecto pequeño. Con ello se valida la hipótesis específica $\mathrm{H}_{1.4}$.

En cuanto a la relación entre el factor defensa de los propios derechos como consumidor y el factor falta de control personal en el uso de las redes sociales, se observa un coeficiente Rho de Spearman de $-.10(\mathrm{p}<.001)$, el cual corresponde a una correlación negativa estadísticamente significativa y que exhibe un tamaño del efecto pequeño. Estos datos permiten señalar que se valida la hipótesis específica $\mathrm{H}_{1.5}$.

En relación con la hipótesis específica $\mathrm{H}_{1.6}$, que plantea la existencia de una relación negativa y estadísticamente significativa entre el factor defensa de los propios derechos como consumidor y el factor uso excesivo de las redes sociales en los participantes, en la Tabla 3, se observa un coeficiente de correlación de -.10 $(\mathrm{p}<.001)$. Este permite señalar que se valida la hipótesis específica $\mathrm{H}_{1.6}$.

Los resultados de la relación entre el factor expresión de enfado o disconformidad y el factor obsesión por las redes sociales muestran un coeficiente Rho de Spearman de -.21 $(\mathrm{p}<.001)$. Este corresponde a una correlación negativa estadísticamente significativa y a un tamaño del efecto pequeño, lo que permite señalar que se valida la hipótesis específica $\mathrm{H}_{1.7}$.

Igualmente, en los resultados de la hipótesis específica $\mathrm{H}_{1.8}$, que plantea la 
existencia de una relación negativa estadísticamente significativa entre el factor expresión de enfado o disconformidad y el factor falta de control personal en el uso de las redes sociales, se puede observar que se obtiene un coeficiente de correlación de -.21 ( $\mathrm{p}<.001)$, que corresponde a un tamaño del efecto pequeño. Este resultado permite concluir que se valida la hipótesis específica $\mathrm{H}_{1.8}$.

En cuanto a la hipótesis específica $\mathrm{H}_{1.9}$, que plantea la existencia de una relación negativa y estadísticamente significativa entre el factor expresión de enfado o disconformidad y el factor uso excesivo de las redes sociales, en la Tabla 5 se observa un coeficiente de correlación de -.19 ( $\mathrm{p}<.001)$, que corresponde a un tamaño del efecto pequeño. Estos datos permiten señalar que se valida la hipótesis específica $\mathrm{H}_{1.9}$.

Por otro lado, en los resultados de la hipótesis específica $\mathrm{H}_{1.10}$, que plantea la existencia de una relación negativa y estadísticamente significativa entre el factor decir "no" y cortar interacciones y el factor obsesión por las redes sociales, se puede observar que se obtiene un coeficiente de correlación de $-.20(\mathrm{p}<.001)$, que corresponde a un tamaño del efecto pequeño. Así, se puede concluir que se valida la hipótesis específica $\mathrm{H}_{1.10}$.

En cuanto a la hipótesis específica $\mathrm{H}_{1.11}$, que plantea la existencia de una relación negativa y estadísticamente significativa entre el factor decir "no" y cortar interacciones y el factor falta de control personal en el uso de las redes sociales, en la Tabla 6 , se observa un coeficiente de correlación de -.19 ( $\mathrm{p}<.001$ ), que corresponde a un tamaño del efecto pequeño. Este resultado valida la hipótesis específica $\mathrm{H}_{1.11}$.

También se puede observar que, entre el factor decir "no" y cortar interacciones y el factor uso excesivo de las redes sociales en los participantes, se obtiene un coeficiente de -.19 ( $p<.001)$, que es negativo, estadísticamente significativo y tiene un tamaño del efecto pequeño. Por tanto, se puede indicar que se valida la hipótesis específica $\mathrm{H}_{1.12}$.

En la correlación Rho de Spearman entre los puntajes del factor hacer peticiones y el factor obsesión por las redes sociales en los estudiantes de una universidad pública y una particular de Lima Metropolitana, se puede apreciar un coeficiente de $-.07(\mathrm{p}<.01)$, lo que indica la existencia de una correlación negativa, estadísticamente significativa y un tamaño del efecto nulo entre ambos factores. Con ello, se puede señalar que se valida la hipótesis específica $\mathrm{H}_{1.13}$.

En cuanto a la relación entre el factor hacer peticiones y el factor falta de control personal en el uso de las redes sociales (Tabla 7), se observa un coeficiente Rho de Spearman de -.03 ( $p>.05$ ), el cual corresponde a una correlación negativa que no es estadísticamente significativa y cuyo tamaño del efecto es nulo. Estos datos permiten señalar que no se valida la hipótesis específica $\mathrm{H}_{1.14}$, que plantea una relación negativa y estadísticamente significativa entre ambos factores.

En relación con la hipótesis específica $\mathrm{H}_{1.15}$, que plantea la existencia de una relación negativa y estadísticamente 
significativa entre el factor hacer peticiones y el factor uso excesivo de las redes sociales en los participantes, se observa un coeficiente de correlación de $-.02(\mathrm{p}>$ $.05)$. Este permite señalar que no se valida la hipótesis específica $\mathrm{H}_{1.15}$.

Los resultados de la correlación entre el factor iniciar interacciones positivas con el sexo opuesto y el factor obsesión por las redes sociales muestran un coeficiente Rho de Spearman de -.19 ( $\mathrm{p}<.001)$, una correlación negativa, estadísticamente significativa y que presenta un tamaño del efecto pequeño. Por tanto, se valida la hipótesis específica $\mathrm{H}_{1.16}$.

Del mismo modo, se presentan los resultados de la hipótesis específica $\mathrm{H}_{1.17}$, que plantea la existencia de una relación negativa y estadísticamente significativa entre el factor iniciar interacciones positivas con el sexo opuesto y el factor falta de control personal en el uso de las redes sociales. En ellos, se puede observar que se obtiene un coeficiente de correlación de -.17 ( $\mathrm{p}<.001)$, lo cual permite concluir que se valida la hipótesis específica $\mathrm{H}_{1.17}$.

En cuanto a la hipótesis específica $\mathrm{H}_{1.18}$, que plantea la existencia de una relación negativa y estadísticamente significativa entre el factor iniciar interacciones positivas con el sexo opuesto y el factor uso excesivo de las redes sociales, en la Tabla 3 , se observa un coeficiente de correlación de $-.13(\mathrm{p}<.001)$. Este permite señalar que se valida la hipótesis específica $\mathrm{H}_{1.18}$.

Tabla 3

Coeficientes Rho de Spearman entre los factores del EHS y los factores del ARS en los estudiantes de una universidad pública y una particular de Lima Metropolitana

\begin{tabular}{lccc}
\hline & $\begin{array}{c}\text { Obsesión } \\
\text { por las redes } \\
\text { sociales }\end{array}$ & $\begin{array}{c}\text { Falta de control } \\
\text { personal en el } \\
\text { uso de las redes } \\
\text { sociales }\end{array}$ & $\begin{array}{c}\text { Uso excesivo } \\
\text { de las redes } \\
\text { sociales }\end{array}$ \\
\hline $\begin{array}{l}\text { Autoexpresión en situaciones } \\
\text { sociales }\end{array}$ & $-.25^{* * *}$ & $-.25^{* * *}$ & $-.20^{* * *}$ \\
\hline $\begin{array}{l}\text { Defensa de los propios } \\
\text { derechos como consumidor }\end{array}$ & $-.12^{* * *}$ & $-.10^{* * *}$ & $-.10^{* * *}$ \\
\hline $\begin{array}{l}\text { Expresión de enfado o } \\
\text { disconformidad }\end{array}$ & $-.21^{* * *}$ & $-.21^{* * *}$ & $-.19^{* * *}$ \\
\hline $\begin{array}{l}\text { Decir "no" y cortar } \\
\text { interacciones }\end{array}$ & $-.20^{* * *}$ & $-.19^{* * *}$ & $-.19^{* * *}$ \\
\hline $\begin{array}{l}\text { Hacer peticiones } \\
\text { Iniciar interacciones positivas } \\
\text { con el sexo opuesto }\end{array}$ & $-.07^{* *}$ & -.03 & -.02 \\
\hline
\end{tabular}

Nota: $\mathrm{N}=1405 .{ }^{* * *} \mathrm{p}<.001$ 


\section{DISCUSIÓN}

Al relacionar el puntaje total de las habilidades sociales y el puntaje total del uso de redes virtuales en estudiantes de una universidad pública y una privada de Lima Metropolitana, se obtuvo una correlación inversa y estadísticamente significativa (Tabla 2), lo cual corrobora lo planteado teóricamente por Gismero (2000); Lucena, Fernández y Francisco (2002); Orihuela (2008); Carballo, Pérez-Jover, Espada, Orgilés y Piqueras (2012), quienes señalan que las personas que tienen adecuadamente desarrolladas sus habilidades sociales son capaces de establecer relaciones interpersonales satisfactorias y pueden llevar a cabo diversos tipos de actividades en su medio social.

En cambio, la proliferación del uso de las redes sociales ha desplazado de manera progresiva y notoria el contacto personal, lo que puede afectar las relaciones interpersonales, pues a través de las redes sociales se va perdiendo la capacidad de interpretar el estado de ánimo de la otra persona, no se puede analizar el lenguaje corporal, e inclusive se puede perder la capacidad de ser asertivos al emitir opiniones. Este resultado también coincide con lo reportado por Zywica y Danowski (2008) en su estudio sobre apariencia social en los usuarios de una red social muy conocida, quienes señalan que los usuarios con perfiles introvertidos y con menor autoestima se esforzaban más por verse populares en esta red social que aquellos que tenían perfiles extrovertidos y alta autoestima. Asimismo, concuerda con lo reportado por García del Castillo, Terol, Nieto, Lledo, Sánchez, Aragón y Sitges (2008); Herrera, Pacheco, Palomar y Zavala (2010); Mejía, Paz y Quinteros (2014), quienes encontraron que los estudiantes que usaban internet con menor frecuencia tenían mayores habilidades sociales.

$\mathrm{Al}$ establecer la relación entre el factor autoexpresión en las situaciones sociales y el factor obsesión por las redes sociales en los estudiantes de una universidad pública y una particular de Lima Metropolitana, se encuentra una correlación inversa y estadísticamente significativa Tabla 3), lo cual coincide con lo planteado teóricamente por Gismero (2000); Ramírez (2008, citado en Godoy, 2010); Escurra y Salas (2014), y lo reportado por García del Castillo, Terol, Nieto, Lledo, Sánchez, Aragón y Sitges (2008); Herrera, Pacheco, Palomar y Zavala (2010), pues los estudiantes que son capaces de expresar sus opiniones, sus sentimientos, intereses, que pueden formular preguntas de manera espontánea y sin sentir ansiedad en diversas situaciones sociales, no piensan constantemente ni están fantaseando con las redes sociales, y no se sienten ansiosos si no pueden acceder a ellas.

También se encontró una relación inversa y estadísticamente significativa entre el factor autoexpresión en situaciones sociales y el factor falta de control personal en el uso de las redes sociales en los participantes (Tabla 3), lo cual coincide con el planteamiento teórico de Gismero (2000); Ramírez (2008, citado en Godoy, 2010); Orihuela (2008); Carballo, 
Pérez-Jover, Espada, Orgilés y Piqueras (2012); Escurra y Salas (2014), así como con las de investigaciones de García del Castillo, Terol, Nieto, Lledo, Sánchez, Aragón y Sitges (2008); Herrera, Pacheco, Palomar y Zavala (2010); Mejía, Paz y Quinteros (2014), porque el estudiante que no siente ansiedad para expresarse en diversas situaciones sociales (alto puntaje en el factor autoexpresión en situaciones sociales) no se descuida en sus tareas ni estudios por no poder controlar el tiempo que utiliza en las redes sociales (bajo puntaje en el factor falta de control en el uso de las redes sociales).

Asimismo, los resultados muestran una relación inversa y estadísticamente significativa entre el factor autoexpresión en situaciones sociales y el factor uso excesivo de las redes sociales (Tabla 3), que coincide con lo planteado teóricamente por Gismero (2000); Lucena, Fernández y Francisco (2002); Ramírez (2008, citado en Godoy, 2010); Carballo, Pérez-Jover, Espada, Orgilés y Piqueras (2012); Escurra y Salas (2014), y con los resultados de investigaciones como las de Valkenburg y Peter (2008); Subrahmanyan y Greenfield (2008); Herrera, Pacheco, Palomar y Zavala (2010); Mejía, Paz y Quinteros (2014), porque los estudiantes con alta autoexpresión en situaciones sociales no presentan dificultades para controlar el uso de las redes sociales ni el tiempo que las utilizan.

En cuanto a la relación entre el factor defensa de los propios derechos como consumidor y el factor obsesión por las redes sociales en los estudiantes de una universidad pública y una particular de Lima
Metropolitana (Tabla 3), se observa una relación inversa y estadísticamente significativa que coincide tanto con lo señalado teóricamente por Gismero (2000); Ramírez (2008, citado en Godoy, 2010), Orihuela (2008); Escurra y Salas (2014) como con los resultados de trabajos empíricos como los de Zywica y Danowski (2008), García del Castillo, Terol, Nieto, Lledo, Sánchez, Aragón y Sitges (2008); Herrera, Pacheco, Palomar y Zavala (2010), pues los estudiantes que son asertivos para defender sus derechos en situaciones de consumo no se sienten comprometidos mentalmente con las redes sociales ni se angustian cuando no pueden acceder a ellas.

Asimismo, se encontró una relación inversa y estadísticamente significativa entre el factor defensa de los propios derechos como consumidor y el factor falta de control personal en el uso de las redes sociales (Tabla 3), coincidente con lo planteado teóricamente por Gismero (2000), Lucena, Fernández y Francisco (2002); Carballo, Pérez-Jover, Espada, Orgilés y Piqueras (2012); Escurra y Salas (2014), así como con lo reportado por Valkenburg y Peter (2008); Subrahmanyan y Greenfield (2008); Herrera, Pacheco, Palomar y Zavala (2010); Mejía, Paz y Quinteros (2014), debido a que los participantes que obtienen puntajes altos en el factor defensa de los propios derechos como consumidor no presentan falta de control en el uso de las redes sociales y pueden interrumpir su uso sin mayores inconvenientes.

Los resultados en la tabla 3 también muestran una correlación inversa y estadísticamente significativa entre el factor 
defensa de los propios derechos como consumidor y el factor uso excesivo de las redes sociales en los participantes, que concuerda con lo planteado teóricamente por Gismero (2000); Orihuela (2008); Escurra y Salas (2014), y con los resultados de estudios empíricos como los de García del Castillo, Terol, Nieto, Lledo, Sánchez, Aragón y Sitges (2008); Herrera, Pacheco, Palomar y Zavala (2010); Mejía, Paz y Quinteros (2014), pues los estudiantes con bajo nivel de asertividad ante desconocidos para defender sus derechos presentan dificultades para poder controlar el uso de las redes sociales y no son capaces de disminuir el tiempo que las utilizan.

En lo que se refiere a la relación entre el factor expresión de enfado o disconformidad y el factor obsesión por las redes sociales (Tabla 3), se observa una relación inversa y estadísticamente significativa, que coincide tanto con lo señalado teóricamente por Gismero (2000); Ramírez (2008, citado en Godoy, 2010); Escurra y Salas (2014) como con los resultados de investigaciones como las de Zywica y Danowski (2008); García del Castillo, Terol, Nieto, Lledo, Sánchez, Aragón y Sitges (2008); Herrera, Pacheco, Palomar y Zavala (2010); Mejía, Paz y Quinteros (2014), pues los estudiantes que puntúan alto en el factor expresión de enfado o disconformidad son personas capaces de enfrentar conflictos y confrontaciones con otros, y puntúan bajo en el factor obsesión por las redes sociales; por lo tanto, no se encuentran comprometidas mentalmente con las redes sociales, no están pensando en ellas constantemente y no se sienten ansiosas si no pueden acceder a ellas.
Asimismo, en la Tabla 3, se encontró una correlación inversa y estadísticamente significativa entre el factor expresión de enfado o disconformidad y el factor falta de control personal en el uso de las redes sociales, resultados que coinciden con lo señalado teóricamente por Gismero (2000); Orihuela (2008); Carballo, Pérez-Jover, Espada, Orgilés y Piqueras (2012); Escurra y Salas (2014), así como con los resultados de estudios como los de Valkenburg y Peter (2008); Subrahmanyan y Greenfield (2008); Herrera, Pacheco, Palomar y Zavala (2010); Mejía, Paz y Quinteros (2014), pues los estudiantes que pueden enfrentar conflictos y confrontaciones con otras personas, que son capaces de expresar que están mortificados o que tienen sentimientos negativos justificados, que pueden expresar su desacuerdo con los demás, no tienen problemas para controlar el uso de las redes sociales; por ello, no se descuidan de sus tareas o sus estudios.

Los resultados de la Tabla 3 también permiten observar una correlación inversa y estadísticamente significativa entre el factor expresión de enfado o disconformidad y el factor uso excesivo de las redes sociales, que concuerda tanto con los planteamientos teóricos de Gismero (2000); Lucena, Fernández y Francisco (2002); Ramírez (2008, citado en Godoy, 2010); Escurra y Salas (2014) como con los resultados de trabajos de investigadores como García del Castillo, Terol, Nieto, Lledo, Sánchez, Aragón y Sitges (2008); Herrera, Pacheco, Palomar y Zavala (2010); Mejía, Paz y Quinteros (2014), pues los estudiantes que no son capaces de expresar su 
enfado, sus sentimientos negativos justificados, su desacuerdo con otras personas, que no pueden enfrentar conflictos, tienden a presentar dificultades para controlar el tiempo que utilizan las redes sociales o las utilizan de manera excesiva.

$\mathrm{Al}$ establecer la relación entre el factor decir "no" y cortar interacciones y el factor obsesión por las redes sociales en los estudiantes de una universidad pública y una particular de Lima Metropolitana, se encuentra una correlación inversa y estadísticamente significativa (Tabla 3), lo cual coincide con lo planteado teóricamente por Gismero (2000); Ramírez (2008, citado en Godoy, 2010); Escurra y Salas (2014), y lo reportado por García del Castillo, Terol, Nieto, Lledo, Sánchez, Aragón y Sitges (2008); Herrera, Pacheco, Palomar y Zavala (2010), pues los estudiantes que son capaces de terminar interacciones que no desean mantener, que son capaces de no prestar algo o no hacer algo que no desean, no piensan constantemente ni están fantaseando con las redes sociales y no les genera ansiedad el no poder acceder a ellas.

También se encontró una relación inversa y estadísticamente significativa entre el factor decir "no" y cortar interacciones y el factor falta de control personal en el uso de las redes sociales en los participantes (Tabla 3), lo cual también coincide con el planteamiento teórico de Gismero (2000); Lucena, Fernández y Francisco (2002); Orihuela (2008); Carballo, Pérez-Jover, Espada, Orgilés y Piqueras (2012); Escurra y Salas (2014), así como con las investigaciones de
García del Castillo, Terol, Nieto, Lledo, Sánchez, Aragón y Sitges (2008); Herrera, Pacheco, Palomar y Zavala (2010); Mejía, Paz y Quinteros (2014), porque el estudiante que es capaz de terminar interacciones que no desea mantener, y que se niega a prestar algo o hacer algo que no desea (alto puntaje en el factor decir "no" $y$ cortar interacciones), no tiene dificultades en sus tareas y estudios por no poder controlar el tiempo que utiliza las redes sociales (bajo puntaje en el factor falta de control en el uso de las redes sociales).

Asimismo, los resultados muestran una relación inversa y estadísticamente significativa entre el factor decir "no" $y$ cortar interacciones y el factor uso excesivo de las redes sociales (Tabla 3), que coincide con lo planteado teóricamente por Gismero (2000); Ramírez (2008, citado en Godoy, 2010); Carballo, Pérez-Jover, Espada, Orgilés y Piqueras (2012); Escurra y Salas (2014), y con los resultados de investigaciones como las de Valkenburg y Peter (2008); Subrahmanyan y Greenfield (2008); Herrera, Pacheco, Palomar y Zavala (2010); Mejía, Paz y Quinteros (2014), porque los estudiantes con baja habilidad para dar por terminadas interacciones que no desean, que no son capaces de negarse a prestar algo o que no pueden negarse a hacer algo que no desean, presentan dificultades para controlar el uso de las redes sociales o no pueden controlar el tiempo que las utilizan.

En cuanto a la relación entre el factor hacer peticiones y el factor obsesión por las redes sociales en los estudiantes de una universidad pública y una particular 
de Lima Metropolitana (Tabla 3), se observa una relación inversa y estadísticamente significativa, que coincide tanto con lo señalado teóricamente por Gismero (2000); Ramírez (2008, citado en Godoy, 2010); Lucena, Fernández y Francisco (2002); Escurra y Salas (2014) como con los resultados de trabajos empíricos como los de Zywica y Danowski (2008), García del Castillo, Terol, Nieto, Lledo, Sánchez, Aragón y Sitges (2008); Herrera, Pacheco, Palomar y Zavala (2010), pues los estudiantes que poseen la habilidad para iniciar conversaciones, para pedir una cita, que pueen decirle un cumplido a otra persona que les resulta atractiva, no se sienten comprometidos mentalmente con las redes sociales ni se angustian al no poder acceder a ellas.

En lo que se refiere a la relación entre el factor iniciar interacciones positivas con el sexo opuesto y el factor obsesión por las redes sociales (Tabla 3 ), se observa una relación inversa y estadísticamente significativa, que coincide tanto con lo señalado teóricamente por Gismero (2000); Ramírez (2008, citado en Godoy, 2010); Escurra y Salas (2014) como con los resultados de investigaciones como las de Zywica y Danowski (2008); García del Castillo, Terol, Nieto, Lledo, Sánchez, Aragón y Sitges (2008); Herrera, Pacheco, Palomar y Zavala (2010); Mejía, Paz y Quinteros (2014), pues los estudiantes que puntúan alto en el factor iniciar interacciones positivas con el sexo opuesto son personas capaces de enfrentar conflictos y confrontaciones con otros, y puntúan bajo en el factor obsesión por las redes sociales; por lo tanto, no se encuentran comprometidas mentalmente con las redes sociales, no están pensando en ellas constantemente ni se sienten ansiosas si no pueden acceder a ellas.

Asimismo, en la Tabla 3, se encontró una correlación inversa y estadísticamente significativa entre el factor iniciar interacciones positivas con el sexo opuesto y el factor falta de control personal en el uso de las redes sociales, resultados que coinciden con lo señalado teóricamente por Gismero (2000); Orihuela (2008); Carballo, Pérez-Jover, Espada, Orgilés y Piqueras (2012); Escurra y Salas (2014), así como con los resultados de estudios como los de Valkenburg y Peter (2008); Subrahmanyan y Greenfield (2008); Herrera, Pacheco, Palomar y Zavala (2010); Mejía, Paz y Quinteros (2014), pues los estudiantes que tienen la habilidad para iniciar conversaciones o pedir una cita, que pueden, de manera espontánea, decir un halago a alguien que les resulta atractivo sin sentirse ansiosos, no presentan problemas para controlar el uso de las redes sociales; por ello, no se descuidan de sus tareas o sus estudios.

Finalmente, los resultados de la Tabla 3 también permiten observar una correlación inversa y estadísticamente significativa entre el factor iniciar interacciones positivas con el sexo opuesto y el factor uso excesivo de las redes sociales, que concuerda tanto con los planteamientos teóricos de Gismero (2000); Ramírez (2008, citado en Godoy, 2010); Escurra y Salas (2014) como con los resultados de trabajos de investigadores como García del Castillo, Terol, Nieto, Lledo, Sánchez, Aragón y Sitges 
(2008); Herrera, Pacheco, Palomar y Zavala (2010); Mejía, Paz y Quinteros (2014), pues los estudiantes que no son capaces de iniciar una conversación, de pedir una cita, que no pueden decir un cumplido sin sentirse ansiosos, tienden a presentar dificultades para controlar el tiempo que utilizan las redes sociales y lo hacen de manera excesiva.

\section{CONCLUSIONES}

Como resultado del estudio, se plantean las siguientes conclusiones:

1. Se encontró una relación inversa y estadísticamente significativa entre las habilidades sociales y el uso de redes sociales virtuales en estudiantes de una universidad pública y una privada de Lima Metropolitana.

2. Se obtuvo una correlación inversa y estadísticamente significativa entre el factor autoexpresión en situaciones sociales y el factor obsesión por las redes sociales en estudiantes de una universidad pública y una privada de Lima Metropolitana.

3. Los resultados muestran una correlación inversa y estadísticamente significativa entre el factor autoexpresión en situaciones sociales y el factor falta de control personal en el uso de las redes sociales.

4. Se obtuvo una correlación inversa y estadísticamente significativa entre el factor autoexpresión en situaciones sociales y el factor uso excesivo de las redes sociales en los participantes.
5. Se encontró una relación inversa y estadísticamente significativa entre el factor defensa de los propios derechos como consumidor y el factor obsesión por las redes sociales en estudiantes de una universidad pública y una privada de Lima Metropolitana.

6. Los resultados muestran una correlación inversa y estadísticamente significativa entre el factor defensa de los propios derechos como consumidor y el factor falta de control personal en el uso de las redes sociales.

7. Se obtuvo una correlación inversa y estadísticamente significativa entre el factor defensa de los propios derechos como consumidor y el factor uso excesivo de las redes sociales en la muestra de estudiantes universitarios.

8. Se encontró una correlación inversa y estadísticamente significativa entre el factor expresión de enfado o disconformidad y el factor obsesión por las redes sociales.

9. Los resultados muestran una correlación inversa y estadísticamente significativa entre el factor expresión de enfado o disconformidad y el factor falta de control personal en el uso de las redes sociales.

10. Los resultados muestran una correlación inversa y estadísticamente significativa entre el factor expresión de enfado o disconformidad y el factor uso excesivo de las redes sociales en los estudiantes universitarios.

11. Se obtuvo una correlación inversa y estadísticamente significativa entre el 
factor decir "no" y cortar interacciones y el factor obsesión por las redes sociales en los participantes.

12. Se encontró una relación inversa y estadísticamente significativa entre el factor decir "no" y cortar interacciones y el factor falta de control personal en el uso de las redes sociales en estudiantes de una universidad pública y una privada de Lima Metropolitana.

13. Los resultados muestran una correlación inversa y estadísticamente significativa entre el factor decir "no" y cortar interacciones y el factor uso excesivo de las redes sociales.

14. Se obtuvo una correlación inversa y estadísticamente significativa entre el factor hacer peticiones y el factor obsesión por las redes sociales en estudiantes de una universidad pública y una privada de Lima Metropolitana.

15. No se obtuvo una correlación inversa y estadísticamente significativa entre el factor hacer peticiones y el factor falta de control personal en el uso de las redes sociales en la muestra de estudiantes universitarios.

16. No se encontró una correlación inversa y estadísticamente significativa entre el factor hacer peticiones y el factor uso excesivo de las redes sociales en los participantes.

17. Los resultados muestran una correlación inversa y estadísticamente significativa entre el factor iniciar interacciones positivas con el sexo opuesto y el factor obsesión por las redes sociales.
18. Los resultados muestran una correlación inversa y estadísticamente significativa entre el factor iniciar interacciones positivas con el sexo opuesto y el factor falta de control personal en el uso de las redes sociales en los estudiantes universitarios.

19. Se obtuvo una correlación inversa y estadísticamente significativa entre el factor iniciar interacciones positivas con el sexo opuesto y el factor uso excesivo de las redes sociales en los participantes.

\section{ReCOMENDACIONES}

a) Implementar programas para prevenir la adicción a redes sociales en estudiantes universitarios

b) Implementar programas de incremento de las habilidades sociales a fin de mejorar la interacción social de los alumnos universitarios

c) Desarrollar estudios similares, pero en diferentes muestras de alumnos, especialmente en alumnos de quinto año de secundaria a fin de establecer si desde esa etapa se empieza a consolidar el uso excesivo de las redes sociales y la manera como afectan las habilidades sociales de los alumnos

d) Desarrollar nuevos estudios, analizando las variables estudiadas, junto con otros constructos psicológicos como la autoeficacia, procrastinación, ansiedad, estrés, entre otros, a fin de identificar los factores que faciliten su desarrollo, mantenimiento y cambio 


\section{REFERENCIAS}

Alarcón, R. (2008). Métodos y diseños de investigación del comportamiento. Lima: Universidad Ricardo Palma.

Ballesteros, R., y Gil, M. D. (2002). Habilidades sociales: evaluación y tratamiento. Madrid: Síntesis.

Carballo, J., Pérez-Jover, M., Espada, J., Orgilés, M., y Piqueras, P. (2012). Propiedades psicométricas de la escala multidimensional de expresión social para la evaluación de habilidades sociales en el contexto de internet. Psicothema, 24(1), 121-126. Recuperado de http://www.psicothema.com/ pdf/3988.pdf

Cohen, J. (1988). Statistical Power Analysis for the Behavioral Sciences. Hillsdale, NY: Lawrence Earlbaum Associates.

Delgado, A. E., Escurra, L. M., y Torres, W. (2006). La medición en psicología y educación: teoría y aplicaciones. Perú: Editorial HOZLO.

Escurra, M., y Salas, E. (2014). Construcción y validación del Cuestionario de Adicción a Redes Sociales (ARS). LIBERABIT, 20(1), 73-91.

Fuente, A., Herrero, J., y Gracia, E. (2010). Internet y apoyo social: sociabilidad online y ajuste psicosocial en la sociedad de la información. Acción Psicológica, 7(1), 9-15.

Galarcio, J. (2006). Formación de la identidad en la adolescencia (Tesis para optar al título profesional de psicólogo). Universidad Pontificia Bolivariana. Medellín. Recuperado de
http://eav.upb.co/banco/files/Tesisidentidadadolescencia.pdf

García del Castillo, J., Terol, M., Nieto, M., Lledo, A., Sánchez, S., Aragón, M., y Sitges, E. (2008). Uso y abuso de internet en jóvenes universitarios. Psiquiatría Interactiva, 20(2), 131-142. Recuperado de http://www.adicciones. es/files/garcia\%20del\%20castillo.pdf

Gismero, E. (2000). Manual de la Escala de Habilidades Sociales, EHS. Madrid: TEA Ediciones.

Godoy, C. (2010). Habilidades sociales en estudiantes de licenciatura en la Facultad de Odontología (Tesis de maestría). Universidad Autónoma de Yucatán, México. Recuperado de Godoy-Celia-MOCE-2010-pdf

Herrera, F., Pacheco M., Palomar, J., y Zavala, D. (2010). La adicción a Facebook relacionada con la baja autoestima, la depresión y la falta de habilidades sociales. Psicología Iberoamericana, 18(1), 6-18. Recuperado de http://www.redalyc.org/src/inicio/ ArtPdfRed.jsp?iCve $=133915936002 \&$ iCveNum015936

Lozares, C. (1996). La teoría de las redes sociales. Papers, 48, 103-126.

Lucena, H., Fernández, D., y Francisco, D. (2002). Diseño de un programa formativo para la mejora de las habilidades sociales en personas mayores. Revista Interuniversitaria, 9, 309-319.

Meattle,J.(2007).Top-50websities-Ranked by Unique Visitors: Digg.com, Facebook, Flickr sky rocketing. Recuperado 
de http://blog.compete/2007/10/30/top50 -websites-domans-digg-youtubeflickr-facebook/

Mejía, G., Paz, J., y Quinteros, D. (2014). Adicción a Facebook y habilidades sociales en estudiantes de una universidad privada de Lima. Revista Cientifica de Ciencias de la Salud, 7(1), 7-14

Orihuela, M. (2008). Internet: la hora de las redes sociales, Nueva Revista, 119, 57-62. Recuperado de http://mccd. udc.es/orihuela/documentos/nueva revista_08.pdf

Razali, N. M., y Wah, Y. B. (2011). Power Comparison of Shapiro-Wilk, Kolmogorov-Smirnov, Lilliefors and Anderson Darling tests. Journal of Statistical Modeling and Analytics, 2(1), 21-33.

Sánchez, H., y Reyes, C. (2015). Metodología y diseños en la investigación cientifica. Lima: Business Support Aneth S.R.L.

Shaffer, D., y Kipp, K. (2007). Psicología del desarrollo: infancia y adolescencia. México: Thompson.
Siegel, S., y Castellan, N. (2012). Estadística no paramétrica. Aplicada a las ciencias de la conducta. México: Trillas

Subrahmanyan, K., y Greenfield, P. (2008). Online Communication and Adolescent Relationships. The future of children, 18(1), 119-148. Recuperado de http:// muse.jhu.edu/login?uri=/journals/future_of_children/v018/18.1.schmidt.html

Valkenburg, P., y Peter, J. (2008). Adolescent's Identity Experiments on the Internet: Consequences for Social Competent and Self-Concept Unity. Communication Research, 35, 208-231. Recuperado de http://crx.sgepub.com/ cgi/content/abstract/35/2/208

Zywica, J., y Danoswki, J. (2008). The Faces of Facebookers ${ }^{\mathrm{TM}}$ : Investigating Social Enhancement and Social Compensation Hyphoteses; Predicting Facebook and Offline Popularity from Sociability and Self-Esteem, and Zapping the Meanings of Popularity with Semantic Networks. Journal of Computer-Mediated Communications, 14, 1-34.Recuperado de http: //www.interscience.wiley.com/journal/121527995/ abstract 
